\title{
Modular Building Blocks for the Development of AUVs - from MARES to TriMARES
}

\author{
Nuno A. Cruz, Aníbal C. Matos and Bruno M. Ferreira \\ INESC TEC, Faculty of Engineering, University of Porto \\ Rua Dr. Roberto Frias, 378 \\ 4200-465 Porto; Portugal \\ nacruz@fe.up.pt
}

\begin{abstract}
The design of an Autonomous Underwater Vehicle (AUV) is governed by a complex tradeoff between mission performance and required payload sensors, and taking into account possible constraints in fabrication, assembly and operational logistics. On a commercial level, the technology is relatively mature, with several companies offering off-the-shelf AUV solutions in a wide range of sizes and performance levels, for a wide variety of operational scenarios. However, to ensure proper performance in specific applications, such broad-range systems require factory customization, with the consequent impact in time and cost. This paper describes a program for the development of underwater vehicles based on modular building blocks. In this case, modularity encompasses both physical parts and also software and control systems. These modules can be rearranged, replaced or individually redesigned to yield a great variety of AUV configurations in a relatively short time. The paper describes the development of MARES, a small hovering AUV, and also TriMARES, a custom 3-body hybrid AUV/ROV, built from the same modules in little over 6 months.
\end{abstract}

\section{INTRODUCTION}

The utilization of underwater robotic systems to assess multiple aspects of the ocean has increased exponentially in the last decade. A great fraction of these operations have been taking advantage of the versatility of commercially available systems, which is certainly a good option in terms of cost effectiveness, for a great variety of applications. On the other hand, as the users get more demanding, certain scenarios require solutions for which specific developments would be more appropriate. However, this encompasses a great deal of customization effort, typically at the factory, with corresponding increase in delivery time and cost. Such inconveniences can be minimized by following a modular architecture in the development of these systems.

The adoption of modular architectures has been exploited in mature manufacturing processes for a long time, with the realization that such approach yields great benefits in terms of adaptation to new demands from customers and also in terms of product variety, i.e., the diversity of solutions that can be manufactured from the same basic components [1]. Such variety should be methodically considered during the design phase, by a proper analysis of module characteristics and how they affect overall system performance [2]. In terms of AUV design, a good example of modularity is the Gavia AUV, with continuous developments to accommodate newer systems [3], although other examples of modularity can also be seen in relatively large vehicles for the installation of specific high performance sensors [4]. These examples are less often seen in small AUVs, with a few exceptions such as the Starfish, from the National University of Singapore [5].

The robotics unit at INESC TEC, in Porto, Portugal, develops custom solutions for specific engineering problems. Its researchers have been involved in the design, development, and deployment of underwater robotic systems for over 15 years, with the main focus on small, man-portable AUVs. INESC TEC is an interface institute between the academic world and the worlds of industry and services and has established worldwide scientific and industrial partnerships in the robotics domain. The development of modular underwater vehicles is of paramount importance, since they can be tuned to address specific scientific challenges, and also be used for ocean sampling in harsh scenarios. Moreover, the institute can benefit from the expertise of international partners in the development and improvement of specific modules.

In this paper, we describe a consistent program for the development of small size AUVs based on modular building blocks. Our approach relies on modularity both in terms of hardware construction, but also in terms of electronics, software and control. Using these blocks, we have first built the MARES AUV [6], back in 2007, a hovering AUV that has been continuously updated and used in the field in many different configurations. In 2011, the versatility of the system components has been pushed further with the development of TriMARES [7], a 75kg, 3-body hybrid AUV/ROV system, which was developed and delivered in little over 6 months.

The remainder of this paper is organized as follows. Section II describes the building blocks and the way they can be interconnected: mechanical components, onboard electronic systems, software organization and control strategies. Then, section III presents two examples of vehicles assembled from the existing blocks: MARES and TriMARES. Section IV describes the current status of these vehicles and points to some operational results. Finally, section V concludes the paper, with some hints on how the modularity may be further exploited to achieve other configurations in the near future. 


\section{Modular BUILding Blocks}

\section{A. Mechanical}

Probably the most obvious aspect of the modularity of our building blocks comes from the design of the hull sections. In order to assemble AUVs from modular components and achieve an overall smooth profile, the blocks have matching edges and constant cross sections. In our case, we have chosen to design modules with $200 \mathrm{~mm}$ of outer diameter. This dimension is a good tradeoff between a small enough size to result in a manageable vehicle and a large enough size to accommodate electronics in dry compartments and to support a wide range of wet sensors. Furthermore, there are several options of materials to be machined with this dimension, from several types of plastics to most common metals, available off the shelf both as rods or tubes. Most of the our parts were machined from polyacetal copolymer in a local machine shop, with some others in aluminium, stainless steel and fiberglass. Polyacetal is a high performance polymer, with a high degree of rigidity and mechanical strength that makes it an excellent weight-saving metal replacement. It is completely corrosion proof and it is readily available at reasonable prices.

All mechanical parts are designed using Solidworks ${ }^{\circledR}$ CAD software, which is essential to progress with the overall design and virtually assemble different components while waiting for part delivery, which can take a very long time for many underwater equipment. Furthermore, the software produces very accurate physical data (weight, centers of mass and buoyancy, etc.) which are critical for the design of motion controllers.

The modules are designed with an identical male/female coupling system, following a sectional modular architecture [1]. They are fixed in place by radial set screws, equally distributed along the perimeter to enable rotation.

Our modules are divided in three different classes:

1) Dry compartments: Cylindrical housings designed to withstand hydrostatic pressure.

Typically, an AUV has a single dry compartment, and the length is chosen to accommodate all computer systems, electronic boards and battery packs. Inside the cylinder, a longitudinal frame eases the task of installing boards and batteries. In a mono-hull design, such batteries are located at the bottom to lower the center of mass and hence increase stability. Since the dry compartment is usually the main source of vehicle buoyancy, it is also possible to extend its length in order to achieve the goal of overall neutral buoyancy. Naturally, such an extension reduces the depth rating of the hull.

Both ends of the cylinders are terminated with end caps, where bore holes are pre-drilled for standard underwater bulkheads or penetrators. A vacuum port allows for leak detection when the housing is sealed, or to exhaust any gas build up during battery charging. Both end caps have a male termination, and the rest of the vehicle can be assembled by attaching any extension modules to both ends (fig. 1).

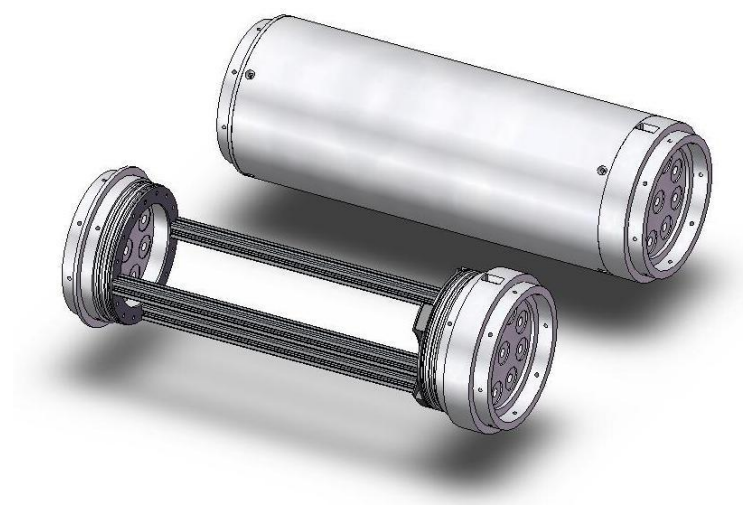

Fig. 1. 3D models of dry compartments, showing the internal frame. Both end caps have a male terminations to accept other modules.

2) Wet extensions: Assortment of water flooded cylindrical sections.

The extensions have a male termination at one end and a female termination at the other, so that they can be stacked, interchanged, or attached directly to the dry compartments, resulting in a variety of possible combinations. Wet extensions can be used with different functionalities, depending on the devices installed:

- Through-hull thrusters for vertical or lateral motion;

- Communication devices, such as radio modems or wifi;

- Navigation devices, such as GPS receivers and acoustic transducers;

- Scientific payload, including various types of sensors (cameras, echo sounders, CTD or optical sensors);

- Mechanical reinforcement rings, for example to provide lifting points;

- Simple hollow sections to provide additional flotation with syntactic foam.

Once these extensions are stacked together, all cabling from the underwater devices pass through the middle gaps to connect to the end caps of the dry compartment.

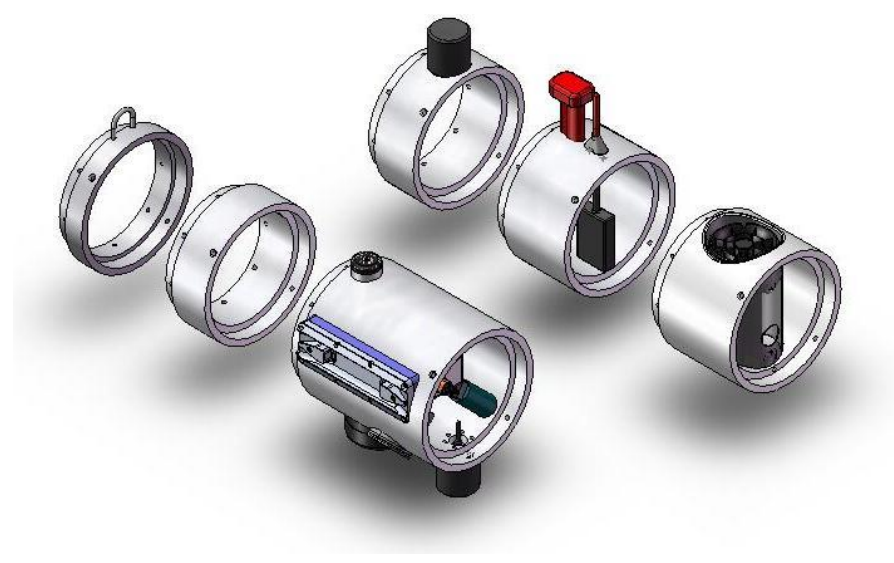

Fig. 2. Examples of water flooded extensions. 
3) End sections: Ellipsoidal shaped, water flooded nose cones and tails, providing the bow and the stern of the AUV.

The tail cones support multiple configurations of propulsion thrusters, that can be rearranged depending on power requirement and maneuverability. They are typically filled with flotation material to compensate for the thruster weight.

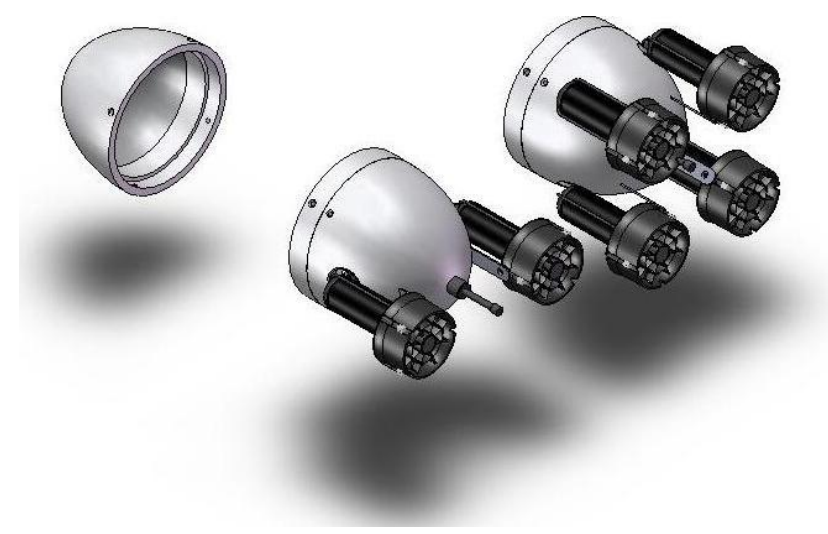

Fig. 3. Examples of end sections, showing a simple nose cone and different types of thruster assemblies. Note the dummy plug in the middle of the thrusters, used to charge the batteries (through and extension cable) once the vehicle is recovered.

Apart from these modules, some other specific parts are needed to assemble an AUV. These are usually small sensor supports, both dry and wet, which we've machined from a variety of materials, or printed directly from the CAD software using a 3-D printer. Finally, a set of syntactic foam parts, with a density of $200 \mathrm{~kg} / \mathrm{m}^{3}$, were also machined and inserted in wet compartments to ensure the weight trimming of the final solution, providing a buoyancy close to neutral.

\section{B. Electrical}

The core of an AUV electrical system relies on a power management module and a computational system. The power management subsystem deals with the conversion, distribution and monitoring of energy to all electrical devices, following a simple architecture (Fig. 4). The rechargeable batteries are installed with the corresponding monitoring boards to measure charge and discharge rates, and charge level. The battery voltage is then converted to the levels required for the onboard devices, and distributed as power busses.

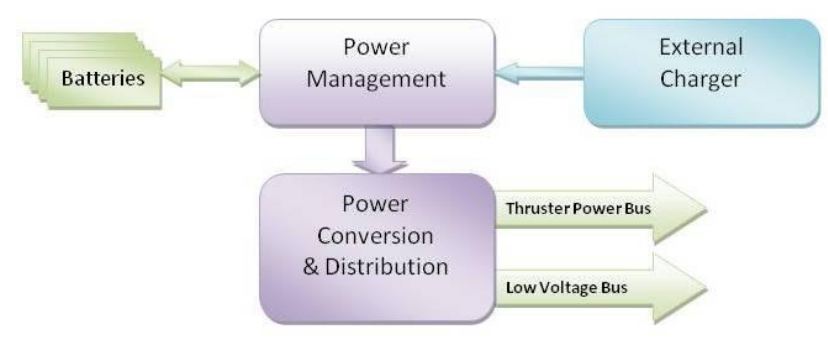

Fig. 4. Overview of on-board power management.
Our onboard computational systems are based on PC104 stacks, with a power supply board, a main processor board, and additional boards to interface with peripherals, such as health monitoring systems, actuation devices, and navigation and payload sensors. A flash disk is used to store both the onboard software and also the data collected during operations. There are many vendors supporting PC104 compatible products, with continuous releases of new boards. Although the PC104 architecture is inherently modular, some special care has to be taken to ensure that the modules installed onboard are fully compatible.

\section{On-board Software}

The on board software runs was developed in $\mathrm{C}++$ and runs on a Linux kernel, with both the operating system and the on board software stored in solid state disk. Each software suite is composed by a set of independent modules (CPU processes) which exchange data using a UDP based message passing mechanism [6], [8]. These modules are hierarchically organized, with the hardware interface modules at the lowest level (Fig. 5). On top of those, two modules - navigation and control - are responsible to provide in real time the position and attitude estimates of the vehicle and to implement the control laws that ensure the adequate behavior of the vehicle, respectively. At the top level, a supervision module is always monitoring the vehicle behavior, scheduling appropriate maneuvers according to a mission plan or to an external command, and taking emergency behavior when required.

Hardware interface modules act as an abstraction layer. Sensory data provided by these modules is transmitted using standard data formats, thus insulating the navigation subsystem from the individual characteristics of the specific devices present in a given configuration of the system. Besides the definition of the data provided by a particular sensor (such as yaw angle, or surge velocity, for example), additional information about the measurements are also taken into account, namely the update rate and a characterization of the measurement error, as well as information related to the relative location and orientation of the sensing device. This makes the whole system highly modular, greatly simplifying the replacement or the inclusion of a new sensor in the system. When that occurs, besides including the corresponding interface module in the onboard computer, it is only required to include in a configuration file the major characteristics of the measurements provided by the sensor, as described above.

The navigation module, based in an extended Kalman filter as described in [6], is prepared to process different measurements - GPS positions at the surface, range measurements to acoustic beacons located in the operation area, velocity data with respect to the water column or to the sea bottom provided by a Doppler velocity logger or any other sensor, accelerations and angular velocities provided by a inertial system, depth data from a pressure cell, or attitude related data from a magnetic compass. The filter internal structure depends on the current sensor package of the vehicle and is automatically defined from the characteristics of the different sensors. 
On the other hand, actuation data transmitted from the control to the hardware interface modules is defined in terms of the forces that each thruster or control surface should produce. This creates a uniform interface between the control module and the low level modules contributing to the independence of the control subsystem with respect to the actuators hardware. Similarly to the case of a new sensor, the inclusion of an actuator in the system (for example, a thruster or a control surface) requires not only the inclusion of the appropriate interface module in the onboard computer but also the standard characterization of the actuator in a proper configuration file. This characterization comprises the location and orientation on the actuator in the vehicle, the limits of actuation, the dependence of the actuation with the velocity of the vehicle and also its maximum update rate and a characterization of the actuation error. These characteristics are then used to the control subsystem to perform thrust allocation and define at each moment the required force from each thruster of control surface. A specific actuation package might impose constraints on the mobility of the vehicle that might turn certain elementary maneuvers unfeasible. Although it is possible to automatically determine the set of feasible forces and torques, and from it evaluate in real time the feasibility of a given elementary maneuver, no automatic process to perform this task in currently implemented in the onboard software. Instead, the set of feasible maneuvers in defined "by hand" for each specific configuration of this class of AUVs, with just an automatic validation by the onboard software.

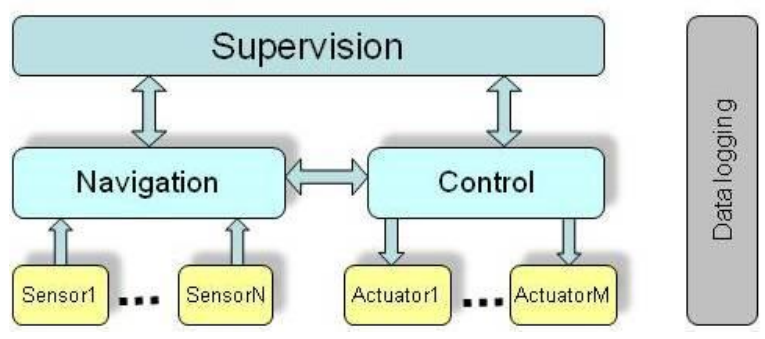

Fig. 5. Overview of on-board software architecture.

\section{Controllers}

The AUV control literature is vast and implements numerous control methods such as model predictive control (MPC), feedback linearization, backstepping, gain-scheduled, linearization and sliding mode. When compared to linear controllers, nonlinear controller for underwater vehicles generally provide superior performances for a broader range of operation. Most of these require a quite precise model of the vehicle dynamics in order to generate the proper thrust and possibly fin angle commands and hence create smooth and precise trajectories. Sliding mode controllers admit larger model errors but at the expense of more abrupt control with natural consequences on trajectories.

Our current approach implements a Lyapounov direct method at the inner loop level. Roughly speaking, this nonlin- ear control method feedforwards the external forces acting on the vehicle body and simultaneously compensate for errors on the velocity. Feedforward is obtained by means of estimation of forces acting on the vehicle as it is impossible to measure them. Such an estimation can use inertial measurements like linear/angular velocities and accelerations. In order to minimize the closed-loop error, the model used for the estimation has to be as precise as possible.

One of the most important aspects in the control of an individual autonomous vehicle is the specification of trajectories. Versatile trajectories and, more generally, behaviors, require the control architecture to include elemental maneuvers. On top of the inner loop velocity controller, which is part of the work presented in [9], a generic control system has been designed so that each robotic platform is able to perform four types of maneuvers, which are briefly described as follows:

- Line-following - The vehicle tracks a line, while keeping a possibly time-varying velocity;

- Circle-following - Given a point and a radius, the vehicle tracks the respective circumference;

- Target tracking (station-keeping) - The vehicle tracks a (possibly time-varying) point and remains stationary;

- Waypoint - This maneuver is concluded once the vehicle reaches a preset position;

In addition to these maneuvers and to reinforce the modularity of the control layer, the controllable degrees-of-freedom (DOF) can be commanded by any external entity. A composition of pose and/or velocity references can be set externally thus allowing to create complex motions without efforts on dynamics control developments. The several controllers intrinsically decouple the several controllable DOFs. Furthermore, the references can be expressed in both the inertial reference frame or the body-fixed one.

Using the above set of maneuvers, any type of complex trajectory can be followed by setting a coherent sequence of instructions. This can be set either by using a static mission script or by instructing the vehicles on-the-fly via a communication link. From the robotics point of view, the maneuvers can be seen as a set of feasible tasks.

The general model of an underwater vehicle is a second order, six-dimensional equation that relates the applied forces and moments with the angular and linear velocities. Inspired by the notation in [10] (see also [11]), the general kinematics and kinetics expressions are respectively given by

$$
\begin{array}{r}
\dot{\eta}=J(\eta) \nu, \\
\dot{\nu}=A(\Theta, \nu) \nu+g(\Theta, \eta)+\tau(\Theta),
\end{array}
$$

where $\eta \in \mathbb{R}^{6}$ is the pose vector, $\nu \in \mathbb{R}^{6}$ is the velocity vector, $J \in \mathbb{R}^{6 \times 6}$ is a matrix that maps the linear and angular velocities expressed in the body-fixed frame into the earthfixed, inertial referential frame. The matrix $A \in \mathbb{R}^{6 \times 6}$ results from the hydrodynamic forces applied on the body of the vehicle when it is moving at a velocity $\nu$. The term $A(\cdot, \cdot) \nu$ constitute the effect of added mass, Coriolis, centripetal and viscous damping forces and moments. The vector $g \in \mathbb{R}^{6}$ 
includes the effects of the restoring forces and moments. The actuation force and moments are given in the vector $\tau \in \mathbb{R}^{6}$, expressed in the body-fixed frame.

Note that the model is parameterized by a set of parameters $\Theta \in\left\{\Theta_{A U V_{1}}, \ldots, \Theta_{A U V_{n}}\right\}$ that are specific of each vehicle. This means that the adopted dynamics control laws expressions can be exactly the same for both the vehicles, i.e., for the velocity stabilization. The only concern in implementation is related with the selection of the corresponding set of parameters $\Theta$. We believe that this versatile feature is very important for reconfigurable systems. Moreover, this allows saving a large amount of time when porting the source control code into a new vehicle. Nonetheless, efforts have to applied in hydrodynamics coefficient derivation so that the model is precise enough to meet the desired control performances.

Hydrodynamics coefficient can be obtained either by computational fluid dynamics (CFD) software, empirical and semiempirical formulas or experimental tests. The determination using the first method implies intensive simulation of the vehicle dynamics using simulated particles of water colliding with the vehicle body. The hydrodynamics coefficients can therefore be determined by assessing the forces, the velocities and the accelerations. The derivation of the coefficients based on formulas remains a valid alternative where an extensive calculus exercise has to be carried out to obtain the final model [12]. Both CFD and formula-based models are affected by errors due to very complex dynamic water simulation in the former, and approximations and observation-based formulas in the later. Experiments can provide very precise coefficients but imply the use of specialized tools and sensors such as the ones used in tow tanks [12], and they are usually very expensive.

The MARES and TriMARES hydrodynamics coefficients were derived based on empirical and semi-empirical formulas found in [12]-[15]. See [9] and [16] for the complete list of coefficients. Since both vehicles have the same planes of symmetries, the parameter sets $\Theta_{M}$ and $\Theta_{T}$ have the same number of hydrodynamic coefficients. In order to determine these coefficients, we've considered a set of modules that includes ellipsoidal noses and tails, cylinders and motors. The protuberances are the antennas, the acoustic transducer, the altimeter and the strobe. All these are characterized by their dimensions and their positions in a body-fixed frame. It should be noted that whenever a change on the configuration is made, the model may have to be re-determined, even under a simple swap of modules. It is very likely that such a change may alter the position of the centers of gravity (CG) and/or buoyancy (CB), even if the length or the positions of the protuberance have not changed, which implies a considerable effort on computation. Hence, we have implemented a program that accepts the length of the vehicle, the positions and the dimensions of the protuberances (including the motors), the throughhull dimensions and positions, the $\mathrm{CG}$ and $\mathrm{CB}$ positions as arguments. Therefore, the determination of the hydrodynamic coefficients for any configuration using the modules (of any length) and the protuberances considered is only subject to the definition of relatively small set of parameters.

\section{VEHICLE EXAMPLES}

\section{A. The MARES AUV}

MARES (Modular Autonomous Robot for Environment Sampling) is a small size, torpedo-shaped AUV, developed in 2007 [6]. The main drive for its design was to develop an open architecture system to carry out research activities in underwater robotics. In particular, we were interested in environmental sampling in coastal waters, and so we settled on a 100 meter depth rating. One of the key requirements regarding motion performance was the possibility of hovering in the water column, which is a feature seldom seen in small vehicles.

MARES (Fig. 6) has a single, $60 \mathrm{~cm}$ long dry compartment, where all electronic boards are installed, with the batteries in the bottom to lower the center of gravity. The main cylinder has 9 holes in each end cap, to accommodate standard bulkhead connectors. To simplify the design, this is the only watertight enclosure and therefore all other equipment is waterproof. Attached to each side of the dry compartment, there is an aluminum lifting ring and a vertical through-hull thruster. The other polyacetal sections were designed to carry navigation and communication modules, and to support the installation of wet sensors.

The overall vehicle shape resembles that of a torpedo, with a constant cross section, except for the ellipsoids both at the nose cone and at the tail. This configuration allows for the vehicle length to be easily extended, simply by inserting the corresponding physical section. The central cylinder provides most of the vehicle flotation and it is also possible to increase its length, for example if more batteries are needed.

Typical small-size AUVs use vertical and horizontal fins to adjust heading and pitch, but this requires a minimum forward velocity for the control surfaces to be effective [17], [18]. On MARES, four independent COTS thrusters provide attitude control both in the horizontal and in the vertical plane. Two horizontal thrusters located at the tail control both forward velocity and rotation in the horizontal plane, while the vertical thrusters control vertical velocity and pitch angle. This arrangement permits operations in very confined areas, with virtually independent horizontal and vertical motion at velocities starting at $0 \mathrm{~m} / \mathrm{s}$. This is one of MARES innovations, as it cannot be seen in any AUV of similar size and weight.

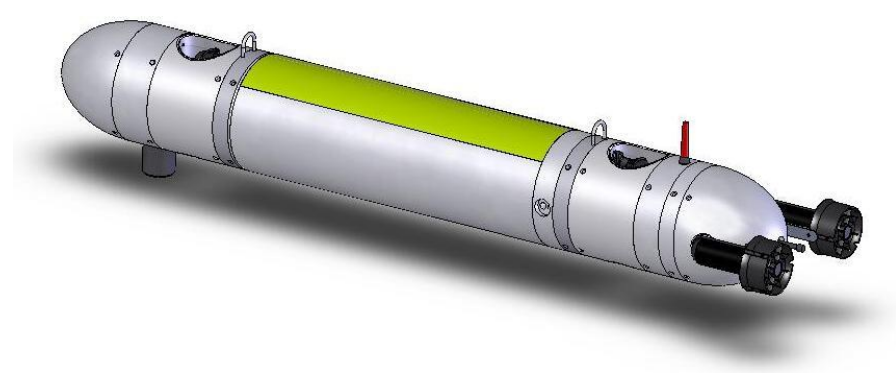

Fig. 6. A 3D CAD model of the first version of MARES, designed in 2006. 
Furthermore, the modularity of the system allows the integration of other thrusters, for example to provide full control of the lateral motion. Naturally, a module with vertical and horizontal fins can also be inserted, to provide more traditional control modes.

Most of the power required by an AUV is spent in propulsion, with only a small amount permanently needed for onboard electronics. In MARES, all energy is stored in rechargeable Li-Ion battery packs, with a total amount of 600 $\mathrm{Wh}$, at $14.4 \mathrm{~V}$. Battery power is directly available to the motor controllers and, through a set of voltage converters, to the rest of the onboard electronics, following a simple distribution as shown in figure 4. Battery endurance greatly depends on vehicle velocity, both in the horizontal and in the vertical plane. For typical horizontal missions, with relatively slow changes in depth, there is sufficient energy for about 8-10 hours of continuous operation (around 20-25 miles or $40 \mathrm{~km}$ ).

\section{B. The TriMARES Hybrid ROV/AUV}

The development of TriMARES followed a contract from a Brazilian consortium, who were interested in a vehicle to monitor the structure of a large dam, and also to assess the quality of the reservoir water [7]. Probably the hardest requirement to meet was the delivery time: about 6 months, which was only possible by taking advantage of all previous designs available for the evolution of MARES and, naturally, with a very tight control on schedule. The other requirements were detailed by the contractor in terms of functionalities and logistics, and can be summarized as:

- Detph rating of 100 meters

- 5 DOF (surge, sway, heave, yaw, pitch)

- Hovering capability

- Forward velocity of at least $1 \mathrm{~m} / \mathrm{s}$

- Absolute position error below 2 meters

- Autonomy of 10 hours

- Prepared for integration of payload sensor package: video and still camera, sonar, water quality sensors

- Hybrid ROV/AUV operation

From an analysis of the requirements and interactions with the contractor, we proposed a multiple body structure, which provides much space for electronics and payload, while still ensuring good hydrodynamics and reduced weight. A similar configuration has been developed more than 20 years ago, with the ABE vehicle at WHOI [19], and recently there have been a few more examples of multiple hull designs, but most of them with much larger weight and dimensions as compared to TriMARES [20]-[22]. The TriMARES' mechanical hull (Fig. 7) is an assembly of three similar bodies linked by a light interconnection structure, which also serves as cable ducting. Each body is built around a $20 \mathrm{~cm}$ diameter, $50 \mathrm{~cm}$ long watertight cylinder, to hold batteries, computers and other electronics. The interface with the external subsystems is done through the end caps, each having 9 holes to accommodate standard bulkhead connectors. Both the cylinders and end caps were machined from polyacetal copolymer (POM) and designed to withstand 100 meters of pressure.

Attached to the end caps, a set of aluminum rings is used to provide lifting points and to hold the bars connecting the 3 bodies, forming a triangular shape with $80 \mathrm{~cm}$ of overall width and $50 \mathrm{~cm}$ of height. This separation is not only physical but also functional. The rechargeable batteries and the power management system are the heaviest part of the vehicle and are located in the bottom cylinder, to lower the center of mass and increase the separation with the center of buoyancy. The top-starboard cylinder holds the main computer, the navigation sensors and the main communication devices. Finally, the payload system is located in the top-port cylinder, with all interfaces for the payload sensors and a second computer to provide realtime processing of sensor information.

All other sections are built with flooded wet extensions, also in POM, with the same outside diameter $(20 \mathrm{~cm})$, therefore ensuring a continuous profile. They are designed to carry wet sensors and thrusters and since they all have common mechanical interfaces, they are fully interchangeable. Finally, each body terminates with ellipsoid-shaped ends, both at the nose cone and at the tail. These are only used as an hydrodynamic shell to reduce vehicle drag and were manufactured in fiberglass from a mould to reduce fabrication cost.

The vehicle required not only a great number of thrusters to ensure maneuverability ( 5 degrees of freedom), but also enough power to overcome the relatively large drag. We've decided to use the same thrusters for all directions, to minimize the number of different parts. From our previous experience, we use small off-the-shelf thrusters from Seabotix, based on brushless DC motors, providing a nominal thrust of $35 \mathrm{~N}$, with possible transients up to $45 \mathrm{~N}$. In order to provide hovering capability, TriMARES has two through-hull vertical thrusters, located in the bottom body. Since they are aligned with a vertical symmetry plane, they can be used to control both heave and pitch simultaneously, with minor influence on the other degrees of freedom. Horizontal propulsion and direction are controlled by four independent thrusters located at the stern, one at the rear end of each of the top bodies, and two in

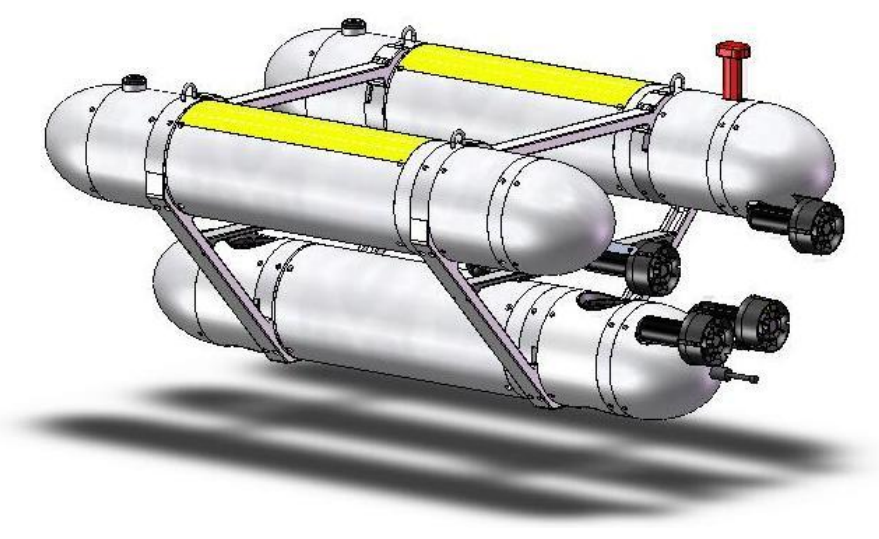

Fig. 7. A 3D CAD model of TriMARES, designed in 2011. 
the lower body. This arrangement enables much more power than required to move the vehicle at 1 meter per second, as desired, but it ensures some degree of redundancy, and allows for a proper operations even in the case of a fault, following the approach in [23]. Finally, a single lateral thruster was also installed to control sway control. This thruster is located close to the center of mass so that the effect in the other degrees of freedom is minimized.

Vehicle energy is provided by rechargeable Li-Ion batteries located in the lower cylinder, with a total energy of $800 \mathrm{Wh}$. The batteries can be fully charged inside the vehicle in approximately 3 hours. A $500 \mathrm{~W}$ DC/DC converter is used to step up the battery voltage level (about $14.6 \mathrm{~V}$ ) into a $28 \mathrm{~V}$ bus needed for the thrusters, therefore all thrusters are connected to the lower cylinder. The raw battery power is also carried to the upper cylinders, where DC/DC converters steps the voltage to the levels required by the various equipment.

TriMARES computational system is composed by two independent computers. The main computer is located in the starboard body and is based on a PC104 stack, with a power supply board, a main processor board (with AMD Geod, LX800 processor at $500 \mathrm{MHz}$ ), and additional boards ts interface with health monitoring systems, actuation devices navigation sensors, and communication systems. The seconc computational system, located in the top-port body, deals witl payload sensors and communicates with the main compute through an ethernet connection. It is also based on a PC104 stack, with a frame grabber to digitize the video from the camera, and the interface with electronics for the sonar transducers. This secondary system can be fully programmed by system users for payload interfacing, processing, and logging, without affecting the normal operation of the modules running in the main computer. This way, system architecture is kept open without putting in risk robustness. When at surface the vehicle can communicate with a shore station using the $\mathrm{WiFi}$ link directly connected to the main computer. Internally, an ethernet switch is connected to both computers. A specially configured network bridge running on the main computer assures a transparent connection between the secondary computer and the shore station through the WiFi link. The switch is also connected to an ethernet optic transceiver to enable communication with the vehicle through a fiber optic umbilical (ROV operation). Although not present in the base version, the system is already prepared for other communication channels, namely a long range UHF radio link at the surface or an acoustic system for underwater communication. In both cases, the link is directly established with the main computer.

\section{VEHICLE OPERATIONS}

The first water tests with MARES were carried out in a water tank. Given that the vehicle has hovering capability, even a small tank is enough to perform many initial tests, from the validation of the main subsystems and ballasting, at the very beginning, to depth control and programming of simple maneuvers and missions. The first sea trials of MARES took place in 2007 (Fig. 8). Since then, MARES has been tested in the field in many different configurations, ranging from 30 to $40 \mathrm{~kg}$ in total weight (with lengths up to about $1.8 \mathrm{~m}$ ). It has been routinely used both as an environmental monitoring platform [24], as a tool for the development of underwater navigation and control ([8]-[10]) or for the demonstration of efficient sampling strategies [25].

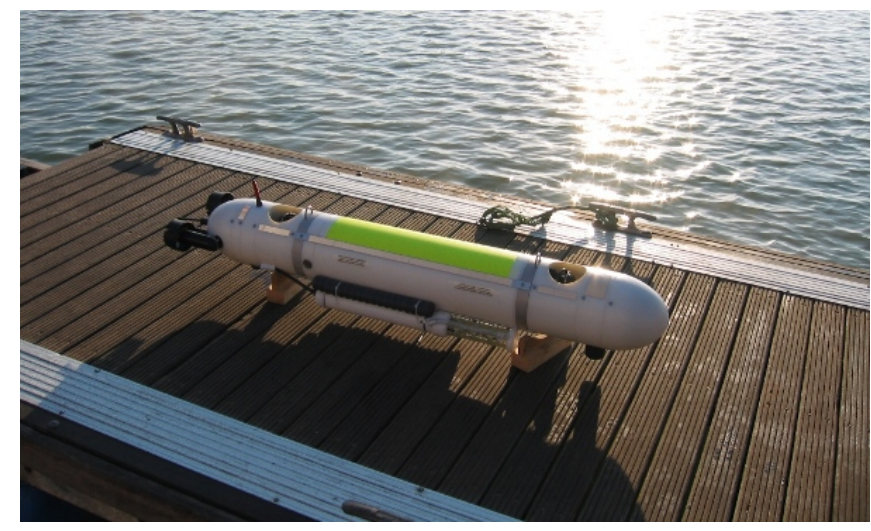

Fig. 8. The MARES AUV ready for sea trials, November 2007.

The first water tests with TriMARES were also carried out in our $5 \mathrm{mx} 5 \mathrm{~m}$ test tank. When we approached the functional version, we moved the test scenario to a reservoir in the Douro river, with a maximum depth of 15 meters. This scenario was chosen not only because it is close to our lab, but also because it is a scaled down version of the final application scenario for TriMARES, in Brazil. During these tests, we have trained the launch and recovery procedure and we have also tested the performance of the combined GPS-WiFi antenna located at the stern of the starboard pod (see figure 9).

Finally, some simple missions were demonstrated, to assess the performance of the motion controllers [16], before the vehicle was shipped to Brazil, in the summer of 2011. Currently, there is an ongoing program of collaboration with Brazilian institutions to provide training and proceed with vehicle development.

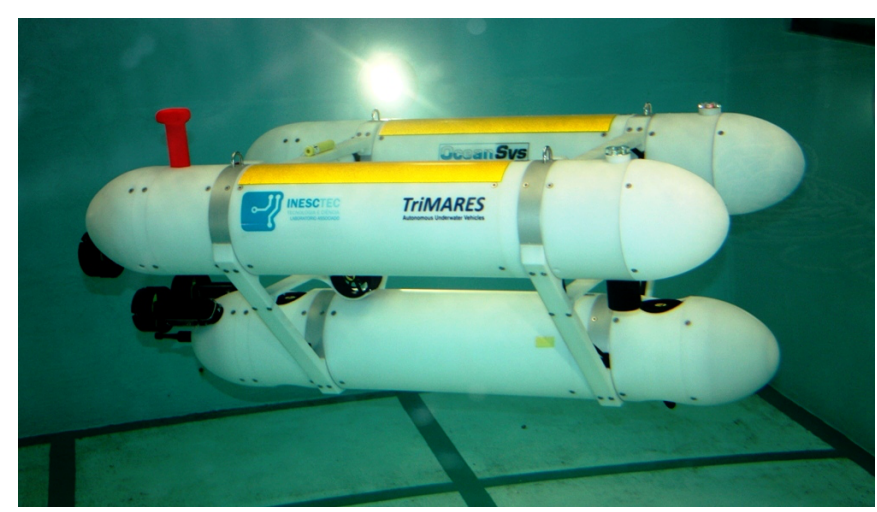

Fig. 9. The TriMARES hybrid AUV/ROV during water trials, June 2011. 


\section{CONCLusion And Future Work}

This paper describes a consistent program for the development of small size AUVs based on modular building blocks. In this case, modularity encompasses both physical parts and also software and control systems. These modules can be rearranged, replaced or individually redesigned to yield a great variety of AUV configurations in a relatively short time. Moreover, given that the development of AUVs requires a coordinated, interdisciplinary work, this modularity ensures a continuous evolution of possible features, without compromising overall reliability of the proven solutions.

Using the modular building blocks, we have first built the MARES AUV, back in 2007, a hovering AUV that has been continuously updated and used in the field in many different configurations. In 2011, after a demand from a Brazilian consortium, the versatility of the system components has gone through a great test - to develop a much larger vehicle to provide extra payload capability, including high quality video, multiple sonars, and water quality sensors. The result was the development of TriMARES, a 75kg, 3-body system hybrid vehicle, with the ability to operate as a standard untethered AUV or as an ROV, using a very thin fiber optic cable to provide real time communications.

The first in-water trials of TriMARES started only 6 months after the final user requirements were discussed. This was only possible due to the reutilizations of many designs already existing during the evolution of MARES. The successful accomplishment of the trials demonstrates that the engineering requirements were met and the reutilization of the available modules contributed to the development of an operational vehicle adequate for the planned tasks.

As for the near future, we think that the versatility of the modular building blocks may be further exploited, to result in a greater variety of vehicles, to address specific scenarios. A 2-body vehicle is a natural extrapolation of the work described in this paper. Other modules can easily be designed to integrate new sensors or actuators, for example to provide control fins to a torpedo AUV. At the same time, the redesign of particular blocks may enlarge the range of potential application scenarios. For example, a redesign of the dry compartment to withstand very high pressures may be used in a deep water AUV, without changes in the other modules.

\section{ACKNOWLEDGMENTS}

This work is funded by the ERDF European Regional Development Fund through the COMPETE Programme (operational programme for competitiveness) and by National Funds through the FCT Fundação para a Ciência e a Tecnologia (Portuguese Foundation for Science and Technology) within project FCOMP - 01-0124-FEDER-022701.

Bruno Ferreira is supported by the Portuguese Foundation for Science and Technology through the $\mathrm{PhD}$ grant SFRH/BD/60522/2009.

\section{REFERENCES}

[1] K. Ulrich, "The role of product architecture in the manufacturing firm," Res. Policy, vol. 24, no. 3, pp. 419-440, May 1995.

[2] M. V. Martin and K. Ishii, "Design for variety: Developing standardized and modularized product platform architectures," Res. Eng. Des., vol. 13, no. 3, pp. 213-235, 2002.

[3] T. Hiller, A. Steingrimsson, and R. Melvin, "Expanding the small auv mission envelope; longer, deeper \& more accurate," in Proc. IEEE/OES Conf. Autonomous Underwater Vehicles AUV'12, Southampton, UK, Sept. 2012.

[4] M. Taylor and A. Wilby, "Design considerations and operational advantages of a modular AUV with synthetic aperture sonar," in Proc. MTS/IEEE Int. Conf. Oceans'11, Kona, HI, USA, Sept. 2011, pp. 1-6.

[5] M. Sangekar, M. Chitre, and T. B. Koay, "Hardware architecture for a modular autonomous underwater vehicle Starfish," in Proc. MTS/IEEE Int. Conf. Oceans'08, Quebec, Canada, Sept. 2008, pp. 1-8.

[6] N. Cruz and A. Matos, "The MARES AUV, a modular autonomous robot for environment sampling," in Proc. MTS/IEEE Int. Conf. Oceans'08, Quebec, Canada, Sept. 2008.

[7] N. A. Cruz, A. C. Matos, R. M. Almeida, B. M. Ferreira, and N. Abreu, "TriMARES - a hybrid AUV/ROV for dam inspection," in Proc. MTS/IEEE Int. Conf. Oceans'11, Kona, HI, USA, Sep. 2011.

[8] A. Matos and N. Cruz, "MARES - navigation, control and on-board software," in Underwater Vehicles, A. V. Inzartsev, Ed. In-Tech, Austria, Jan. 2009, ch. 17, pp. 315-326.

[9] B. Ferreira, A. Matos, N. Cruz, and M. Pinto, "Modeling and control of the MARES autonomous underwater vehicle," Mar. Tech. Soc. J., vol. 44, no. 2, Mar./Apr. 2010.

[10] B. M. Ferreira, J. Jouffroy, A. C. Matos, and N. A. Cruz, "Control and guidance of a hovering AUV pitching up or down," in Proc. MTS/IEEE Int. Conf. Oceans'12, Hampton Roads, VA, USA, Oct. 2012.

[11] T. I. Fossen, Guidance and Control of Ocean Vehicles. UK: John Wiley \& Sons, 1995

[12] T. Prestero, "Verification of a six-degree of freedom simulation model for the REMUS autonomous underwater vehicle," Master's thesis, Massachusetts Institute of Technology and Woods Hole Oceanographic Institution, Sep. 2001.

[13] O. Faltinsen, Hydrodynamics of high-speed marine vehicles. Cambridge University Press, 2005.

[14] F. M. White, Fuid Mechanics, 4th ed. McGraw-Hill, 2002.

[15] S. F. Hoerner, Fluid-Dynamic Drag. Published by author, 1965.

[16] B. M. Ferreira, A. C. Matos, and N. A. Cruz, "Modeling and control of TriMARES AUV," in Proc. 12th Int. Conf. on Autonomous Robot Systems and Competitions, Robotica 2012, E. Bicho, F. Ribeiro, and L. Louro, Eds., Guimares, Portugal, 2012, pp. 57-62.

[17] C. von Alt, B. Allen, T. Austin, and R. Stokey, "Remote environmental measuring units," in Proc. IEEE Symp. Autonomous Underwater Vehicle Tech. AUV'94, Cambridge, MA, USA, Jul. 1994.

[18] J. Crowell, "Small AUV for hydrographic applications," in Proc. MTS/IEEE Int. Conf. Oceans'06, Boston, MA, USA, Sept. 2006.

[19] D. R. Yoerger, A. M. Bradley, and B. B. Walden, "The autonomous benthic explorer (ABE): An AUV optimized for deep seafloor studies," in Proc. 7th Int. Symp. on Unmanned Untethered Submersible Technology, Durham, NH, USA, 1991, pp. 60-70.

[20] H. Kondo, S. Yu, and T. Ura, "Object observation in detail by the AUV "Tri-Dog 1" with laser pointers," in Proc. MTS/IEEE Int. Conf. Oceans'01, Honolulu, HI, USA, Nov. 2001.

[21] D. Shea, C. Williams, M. He, P. Crocker, N. Riggs, and R. Bachmayer, "Design and testing of the Marport SQX-500 twin-pod AUV," in Proc. IEEE/OES Conf. Autonomous Underwater Vehicles AUV 2010, Monterey, CA, USA, Sept. 2010.

[22] D. Ribas, P. Ridao, L. Mag, N. Palomeras, and M. Carreras, "The Girona 500, a multipurpose autonomous underwater vehicle," in Proc. IEEE Int. Conf. Oceans'11, Santander, Spain, June 2011.

[23] B. Ferreira, A. Matos, and N. Cruz, "Automatic reconfiguration and control of the MARES AUV in the presence of a thruster fault," in Proc. IEEE Int. Conf. Oceans'11, Santander, Spain, June 2011.

[24] P. Ramos and N. Abreu, "Using an AUV for assessing wastewater discharges impact: an approach based on geostatistics," Mar. Tech. Soc. J., vol. 45, no. 2, pp. 74-87, Mar./Apr. 2011.

[25] N. Cruz and A. Matos, "Adaptive sampling of thermoclines with autonomous underwater vehicles," in Proc. MTS/IEEE Int. Conf. Oceans'10, Seattle, WA, USA, Sept. 2010. 